Infusionsther Transfusionsmed 1993;20:196-197

\title{
Inhalt, Vol. 20, No. 5, 1993
}

\section{Contents}

Originalarbeiten

Posttransfusionelle Purpura: Klinische und immunvlogische Untersuchungen bei 38 Patientinnen

Kroll, H., Kiefel, V, Mueller-Eckhardt, C 198

Original Paper

Posttransfusion Purpura: Clinical and Immunological Investigations in 38 Patients

Kroll, HL, Kiefel, V, Mueller-Eckhardt, C

198

Safety of Dextran in Relation to Other Colloids -Ten Years Experience with Hapten Inhibition 206 Ljungström, K.-G

Comparative Study of Blood and Infusion Warmers at Different Flow Rates

Die Sicherheit von Dextran im Vergleich mit anderen Kolloiden - zehn Jahre Erfahrung mit

Haptenhemmung

Ljungström, K.-G

Vergleichende Untersuchung von Blut- und Infusions-wärmern bei verschiedenen

Durchflußraten

Dietz, S., Behne, M 212 Dietz, S., Behne, M.

!06

212

Übersichtsarbeit

Physiologie und Klinik des extrazellulären Bikarbonat-Pools: Plädoyer für einen bewußten

Umgang mit $\mathrm{HCO} 3$

Zander, R 217

Review Article

Physiology and Clinical Practice of the Extracellular Bicarbonate Pool: Appeal for Deliberate Handling of $\mathrm{HCO} 3$

Zander, R 217

Briefe an die Herausgeber

236

Letters to the Editors

2 Î6

Gesellschaftsmitteilungen

Society Bulletins 
Mitteilungen der Deutschen Gesellschaft für Transfusions

medizin und Immunhämatologie 241

Reports of the Deutsche Gesellschaft für Transfusions

medizin und Immunhämatologie 241

Band 20, Heft 5, Oktober 1993

Infusionstherapie $\mathrm{u} \prod \mathrm{d}$ Transfusionsmedizin

Inhalt

Contents

Perioperatives Monitoring, Teil 3

Monitoring der Mikrozïrkulation

Monitoring durch Messung des gastrointestinalen Mukosa-pH-Wertes (pHi)

Frey, L., Pacheco, A. M

Perioperative Monitoring, Part 3

Monitoring of Microcirculation

Monitoring by Measuring Gastrointestinal Mucosal $\mathrm{pH}$ Value (pHi)

248 Frey, L, Pacheco, A. M

248

Mikrovaskuläres Monitoring mit Hilfe der Mercury-in-Silastic-Strain-Gauge-Plethysmographie (MSG)

Christ, E, Gartside, LB, Kox,W.J., Gamble, J

Microvascular Monitoring Using the Mercury in Silastic Strain Gauge Plethysmography (MSG)

253 Christ, F, Gartside, LB, Kox, W.J, Gamble, J

253

Zerebrales Monitoring

Cerebral Monitoring

Möglichkeiten der zerebralen Überwachung Approaches in Cerebral Monitoring

Baethmann, A 260 Baethmann, A

260

Stellenwert der transkraniellen Doppler-Sonographie

Schregel, W.

Relevance of Transcranial Doppler Sonography

267 Schregel, W. 267

Monitoring intraoperativer akustischer Reizverarbeitung Monitoring of Intraoperative

Auditory Stimulus Processing

mittels akustisch evozierter Potentiale Using Auditory-Evoked Potentials

Schwender, D, Madler, C, Klasing, S, Pöppel, E, Peter, K 272 Schwender, D, Madler, C,

Klasing, S, Pöppel, E, Peter, K 272

Impressum 195 Imprint195

Inhaltsverzeichnis 196 Contents 196

Industrieforum244 Industrial Forum 244 
Buchbesprechungen 245 Book Reviews 245

Hinweise für Autoren 277 Instructions to Authors

277

Bibliographischer Hinweis: Inhaltsverzeichnisse dieser Zeitschrift erscheinen regelmäßig in current contents ${ }^{\circledR}$ sowie in anderen bibliographischen Diensten. 